\title{
Associations of obesity with antidiabetic medication use after living kidney donation: An analysis of linked national registry and pharmacy fill records
}

\author{
Krista L. Lentine ${ }^{1}$ (D) | Farrukh M. Koraishy ${ }^{1}$ | Nagaraju Sarabu ${ }^{2}$ (D) | Abhijit S. Naik ${ }^{3}$ \\ Ngan N. Lam ${ }^{4}$ | Amit X. Garg ${ }^{5}$ | David Axelrod ${ }^{6}$ (D) | Zidong Zhang ${ }^{1}$ (D) | Gregory \\ P. Hess ${ }^{7}$ (D) | Bertram L. Kasiske ${ }^{8}$ | Dorry L. Segev ${ }^{9}$ | Macey L. Henderson ${ }^{9}$ (D) |

\begin{abstract}
Allan B. Massie $^{9}$ | Courtenay M. Holscher ${ }^{9}$ (i) | Mark A. Schnitzler ${ }^{1}$
${ }^{1}$ Center for Abdominal Transplantation, Saint Louis University School of Medicine, St. Louis, MO, USA

${ }^{2}$ Division of Nephrology, Department of Medicine, University Hospitals Cleveland Medical center, Cleveland, OH, USA

${ }^{3}$ Division of Nephrology, Department of Medicine, University of Michigan, Ann Arbor, MI, USA

${ }^{4}$ Division of Nephrology, Department of Medicine, University of Alberta, Edmonton, AB, Canada

${ }^{5}$ Division of Nephrology, Department of Medicine, Western University, London, ON, Canada

${ }^{6}$ University of lowa Transplant Institute, University of lowa School of Medicine, lowa City, IA, USA

${ }^{7}$ Drexel College of Medicine, Drexel University, Philadelphia, PA, USA

${ }^{8}$ Department of Medicine, Hennepin County Medical Center, Minneapolis, MN, USA

${ }^{9}$ Division of Transplantation, Department of Surgery, Johns Hopkins University, Baltimore, MD, USA
\end{abstract}

\section{Correspondence}

Krista L. Lentine, Center for Abdominal

Transplantation, Saint Louis University, 1402

S. Grand Blvd., St. Louis, MO 63104, USA.

Email: krista.lentine@health.slu.edu

Funding information

This work was conducted under the auspices of the Hennepin Healthcare Research Institute (HHRI), contractor for the Scientific Registry of Transplant Recipients (SRTR), as a deliverable under contract no. HHSH250201500009C (US Department of Health and Human Services, Health Resources and Services Administration, Healthcare Systems Bureau, Division of Transplantation). As a US Governmentsponsored work, there are no restrictions on its use. The interpretation and reporting of these data are the responsibility of the author(s) and in no way should be seen as an official policy of or interpretation by the SRTR or the US Government. Supported in part by a grant from the National Institute of Diabetes and Digestive and Kidney Diseases, R01-DK096008. NNL was supported by a KRESCENT New Investigator Award.

\begin{abstract}
We examined a novel linkage of national US donor registry data with records from a pharmacy claims warehouse (2007-2016) to examine associations (adjusted hazard ratio, ${ }_{L C L} \mathrm{aHR}_{U C L}$ ) of post-donation fills of antidiabetic medications (ADM, insulin or non-insulin agents) with body mass index (BMI) at donation and other demographic and clinical factors. In 28515 living kidney donors (LKDs), incidence of ADM use at 9 years rose in a graded manner with higher baseline BMI: underweight, $0.9 \%$; normal weight, $2.1 \%$; overweight, $3.5 \%$; obese, $8.5 \%$. Obesity was associated with higher risk of ADM use compared with normal BMI (aHR, ${ }_{3.36} 4.59_{6.27}$ ). Metformin was the most commonly used ADM and was filled more often by obese than by normal weight donors (9-year incidence, $6.87 \%$ vs $1.85 \%$, aHR, $3.555^{5.00_{7.04}}$ ). Insulin use was uncommon and did not differ significantly by BMI. Among a subgroup with BMI data at the 1-year post-donation anniversary $(n=19528)$, compared with stable BMI, BMI increase $>0.5 \mathrm{~kg} / \mathrm{m}^{2}$ by year 1 was associated with increased risk of subsequent ADM use (aHR, ${ }_{1.03} 1.48_{2.14}, P=.04$ ). While this study did not assess the impact of donation on the development of obesity, these data support that among LKD, obesity is a strong correlate of ADM use.
\end{abstract}




\section{KEYWORDS}

antidiabetic medications, diabetes mellitus, health outcome, living donation, pharmacy claims, registries

\section{1 | INTRODUCTION}

Medical evaluation of living kidney donor candidates focuses on screening for known risk factors for adverse outcomes, and supporting donors with acceptable risks in informed decision making. ${ }^{1}$ As in the general population, diabetes mellitus has been considered a leading etiology of the uncommon end-stage renal disease (ESRD) events that occur in donors, particularly for ESRD late after donation. In a US registry analysis of 125427 living donors, ESRD beyond 10 years post-donation was predominantly reported as diabetic and hypertensive (later vs early incidence rate ratios, 7.7 and 2.6, respectively). ${ }^{2}$ In a single-center study of 3956 predominantly white living donors, $25 \%$ of ESRD events with known causes ( $n=6 / 25$ ) were related to diabetes. ${ }^{3}$ While pre-donation diabetes is typically considered an exclusion for donation (mandated in current Organ Procurement and Transplantation Network [OPTN] policy), ${ }^{4}$ risk factors for post-donation diabetes mellitus (PDDM), and implications for donor counseling and selection, are not well defined.

Obesity is a well-established risk factor for diabetes and for chronic kidney disease and ESRD in the general population, 5,6 but the outcome implications of obesity in living donors are controversial. A recent meta-analysis based on data from nearly 5 million healthy persons identified from seven general population cohorts, designed to inform donor candidate evaluation, found a modest association of baseline body mass index $(\mathrm{BMI})>30 \mathrm{~kg} / \mathrm{m}^{2}$ with increased risk of ESRD over median cohort follow-up of 4-16 years (adjusted hazard ratio (aHR), $\left.{ }_{1.04} 1.16_{1.29}\right) .^{7}$ A study of 119769 donors from the US found a stronger impact, such that each unit increase in BMI among overweight and obese donors increased the risk of ESRD by $7 \% .{ }^{8}$ In contrast, a single-center study of 3752 predominantly white donors (1994-2016) found that obesity increased the risk of PDDM 3-fold but was not associated with $\operatorname{ESRD}(P=.46) .{ }^{9}$

Uncertainty about the long-term risks of obesity in living donors is reflected by variation in both clinical practice guidelines and clinical practice. Prior guidelines for the evaluation and care of living kidney donor candidates recommend $\mathrm{BMI}>35 \mathrm{~kg} / \mathrm{m}^{2}$ as an absolute or relative contraindication to donation, ${ }^{10-16}$ while other guidelines recommend careful consideration of other comorbid conditions in donor candidates with $\mathrm{BMI}>30 \mathrm{~kg} / \mathrm{m}^{2}{ }^{11,13,17}$ In contrast, based on limited evidence in the donor population, the 2017 Kidney Disease: Improving Global Outcomes "Guideline for the Evaluation and Care of Living Donors" recommends that the decision to approve donor candidates with $\mathrm{BMI}>30 \mathrm{~kg} / \mathrm{m}^{2}$ should be individualized based on demographic and health profile in relation to the transplant program's acceptable risk threshold. ${ }^{1}$ In the context of the national obesity epidemic, overweight, and obese donors now account for two-thirds of all accepted living donors nationally, although there is substantial center-level variation in acceptance practices by BMI, particularly for very obese donors. ${ }^{18}$

At the crossroads of controversy in the implications of obesity in living donor selection, and in recognition of diabetes mellitus as an important cause of ESRD when it occurs in donors, pursuit of better understanding of the implications of pre-donation obesity for comorbidity such as diabetes is warranted. Pharmacy claims offer a non-obtrusive measure of prescribed health care that do not rely on patient self-report and are increasingly used in observational investigations of large populations including transplant and donation-related epidemiologic studies. ${ }^{19-25}$ To this end, we examined a novel linkage of national US transplant registry data with records from a pharmacy claims warehouse that identifies antidiabetic medication (ADM). Our goals were to identify the incidence of ADM use after donation, and to assess variation in risk according to baseline BMI and other demographic and clinical factors.

\section{2 | MATERIALS AND METHODS}

\section{1 | Data sources}

We conducted a retrospective cohort study using linked healthcare databases in the United States. ${ }^{26}$ This study used data from the Scientific Registry of Transplant Recipients (SRTR). The SRTR data system includes data on all donors, waitlisted candidates, and transplant recipients in the United States, submitted by the members of OPTN. The Health Resources and Services Administration (HRSA), US Department of Health and Human Services, provides oversight of the activities of the OPTN and SRTR contractors. The study was approved by the Saint Louis University Institutional Review Board.

Pharmacy fill data were assembled by linking SRTR records for living kidney donors with billing claims from a large US pharmaceutical claims data $(P C D)$ warehouse that maintains prescription drug fill records including self-paid fills and those reimbursed by private and public payers. The PCD comprises National Council for Prescription Drug Program 5.1-format prescription claims aggregated from multiple sources including data clearinghouses, retail pharmacies, and prescription benefit managers for approximately $60 \%$ of US retail pharmacy transactions. Individual claim records include the date of a given pharmacy fill with the National Drug Code identifying agent and dosage. After institutional review board and HRSA approvals, PCD records were linked with SRTR records for living donors.

We applied a deterministic deidentification strategy wherein patient identifiers (last name, first name, sex, date of birth, and ZIP code of residence) were transformed before delivery to the Saint Louis University researchers with Health Information Portability and Accountability Act and HITECH-certified encryption technology. 
The patient deidentification software uses multiple encryption algorithms in succession to guarantee that the resulting "token" containing encrypted patient identifiers can never be decrypted. However, the algorithm yields the same results for a given set of data elements, such that linkages by unique anonymous tokens are possible.

\section{2 | Population and covariates}

We included living kidney donors in the SRTR registry who donated between 2007 and 2016, and had linked pharmacy fill records covering the donation event and post-donation follow-up. Baseline donor demographic and clinical information ascertained from SRTR at the time of donation included: age, sex, race, donor-recipient relationship, health insurance status, BMI, physical limitations, education level, employment status, history of smoking, pre-donation hypertension, pre-donation estimated glomerular filtration rate (eGFR), and type of nephrectomy as reported by the transplant center. Body mass index was categorized according to World Health Organization criteria as follows: underweight, $<18.5$; normal weight, 18.5 to $<25$; overweight, 25 to $<30$; obese, $>30 \mathrm{~kg} / \mathrm{m}^{2}$.

\section{3 | Outcomes}

The primary outcome was time to ADM use as defined by a pharmacy fill dates. Use of insulin and use of non-insulin agents were also examined separately (Table S1). Categories of non-insulin agents were examined in secondary analyses.

\subsection{Statistical analyses}

Data management and analyses were performed with Statistical Analysis Software (SAS) for Windows, version 9.3 (SAS Institute Inc). In all outcome analyses, we interpreted 2-tailed $P$-values less than 0.05 as statistically significant. Distributions of baseline traits among living kidney donors with and without ADM use were compared by the Chi-square test. The cumulative incidence of ADM use was estimated by the Kaplan-Meier method, with a time scale of years since donation, and stratified by BMI categories for comparison. We also estimated the cumulative incidence of insulin use and use of each category of antidiabetic agent by 9 years. Participants were censored at the end of pharmacy data eligibility, death, or end of the study (December 31, 2016). We examined associations ( ${ }_{\mathrm{LCL}} \mathrm{aHR}_{\mathrm{UCL}}$ ) of $\mathrm{BMI}$ at donation and other baseline donor demographic and clinical factors with ADM fills using multivariable Cox regression.

\section{3 | RESULTS}

There were 58601 living donors recorded in the SRTR in the study period. Of these, 28530 had linked PCD data covering the donation event and some follow-up; 321 were excluded for missing BMI data, and 15 were excluded based on indication of pre-donation diabetes in the registry. The mean duration from donation to end of follow-up was 3.8 years (maximum 10.2 years). Among the final study sample of 28515 living donors, the mean age was 42.9 years, $73.7 \%$ were white, nearly $50 \%$ were biologically related to their recipient, $11 \%$ had hypertension, and $96 \%$ of the donor nephrectomies were laparoscopic (Table S1). The baseline characteristics of the study sample were similar to those of all donors recorded in the SRTR database during the same time period (Table S2). The BMI distribution of the study sample included: $<18.5 \mathrm{~kg} / \mathrm{m}^{2}, 0.9 \% ; 18.5$ to $<25 \mathrm{~kg} / \mathrm{m}^{2}$, $33.4 \%$; 25 to $<30 \mathrm{~kg} / \mathrm{m}^{2}, 41.5 \%$; $>30 \mathrm{~kg} / \mathrm{m}^{2}, 23.3 \%$. Obese donors were more likely to be older, men, African American or Hispanic, past or current smokers, to have lower than college education, to have no or unknown insurance status at donation, to donate to a related recipient with diabetes, to be hypertensive, and to have slightly lower pre-donation eGFR (Table 1).

\subsection{Association of obesity with ADM use}

The incidence of ADM use at 9 years rose in a graded manner with higher BMI: underweight, 0.9\%; normal weight, 2.1\%; overweight, 3.5\%; obese, $8.5 \%$ (Figure 1). Compared with donors with normal $\mathrm{BMI}$, after adjustment for age, sex, and race, overweight donors were two times more likely to fill ADM (aHR, $1.632 .24_{3.07}$ ), and obese donors were four times more likely (aHR, ${ }_{3.39} 4.62_{6.30}$ ). These relationships persisted after adjustment for other demographic and clinical factors recorded in the registry (Table 2), such that overweight and obese baseline BMI was associated with 2- and 4-times the likelihood of ADM use, respectively (aHR, ${ }_{1.62} 2.23_{3.06}$ and ${ }_{3.36}{ }^{4.59}{ }_{6.27}$ ). When comparing ADM treatments, these differences were primarily due to non-insulin-based therapies. After covariate adjustment, other significant correlates of ADM included female sex (aHR, $1.66^{2.17}{ }_{2.83}$ ) and more recent years of donation (Table 2). Compared with donations in 2007-2010, donations in 2014-2016 were associated with more than twice the likelihood of ADM use (aHR, $1.60^{2.36_{3.48}}$ ) (Table 2, Figure 2). Donation to a related recipient with diabetes was associated with a trend toward higher risk of ADM use, but this relationship was not statistically significant. Compared with white race, black race and Hispanic ethnicity were not significantly associated with ADM use overall, but were associated with 3.5 and 2.9 times the likelihood of insulin use after donation.

\subsection{Patterns of types of ADM use}

Metformin was the most commonly used ADM after living donation. By 9 years post-donation, use of metformin rose with higher pre-donation BMI: underweight, 0.9\%; normal weight, 1.8\%; overweight, $3.3 \%$; obese, $6.9 \%$ (Figure 3). Use of most classes of diabetes agents was higher in obese donors. Compared with normal BMI, obesity at donation was associated with five times the likelihood of metformin use (aHR, $3.555^{5.00_{7.04}}$ ), three times the likelihood of sulfonylurea use (aHR, $\left.{ }_{1.36} 3.16_{7.34}\right)$, and eight times the likelihood of other agent use (aHR, $2.428 .32_{28.61}$ ) (Figure 4). Obesity at donation was not significantly associated with use of insulin or glitazones, which were both filled infrequently ( $<0.6 \%$ and no fill at 9 years). 
TAB LE 1 Distribution of clinical factors in the study sample of living kidney donors according to body mass index (BMI) at donation

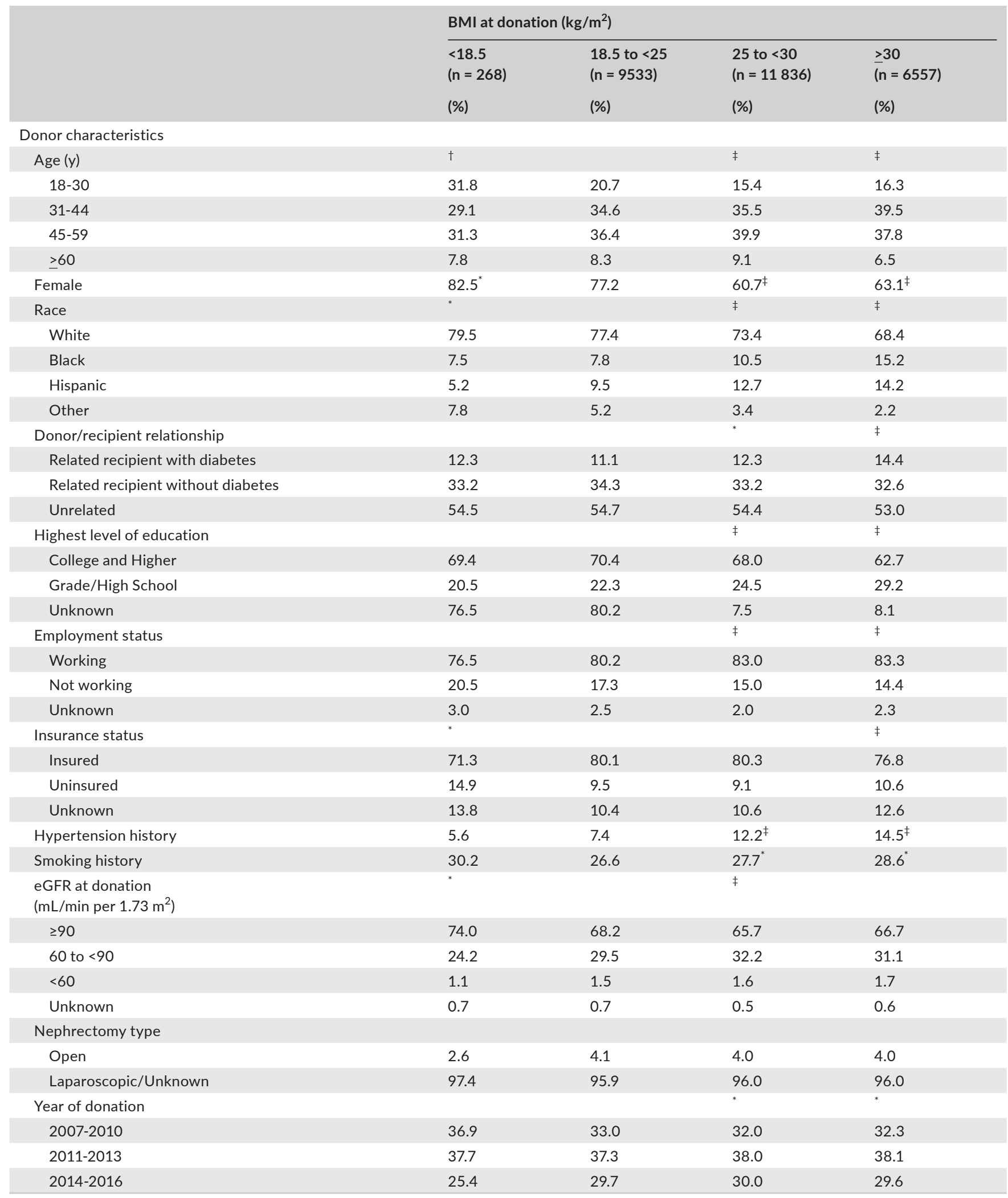

Data presented as percentages (\%) of donors in each BMI category with the indicated baseline trait (column percentages).

ADM, antidiabetic medication; BMI, body mass index; eGFR, estimated glomerular filtration rate.

$\mathrm{P}$-values for variation of distribution of clinical factor according to BMI level at donation:

${ }^{*} P<.05-.002$;

${ }^{\dagger} P=.001-.0002$

${ }^{\ddagger} P<.0001$. 
FIGURE 1 Incidence of antidiabetic medication use according to body mass index (BMI) at donation. A, Any antidiabetic medication use. B, Noninsulin antidiabetic medication use. ${ }^{\ddagger} P<.0001$ compared with reference (Ref)
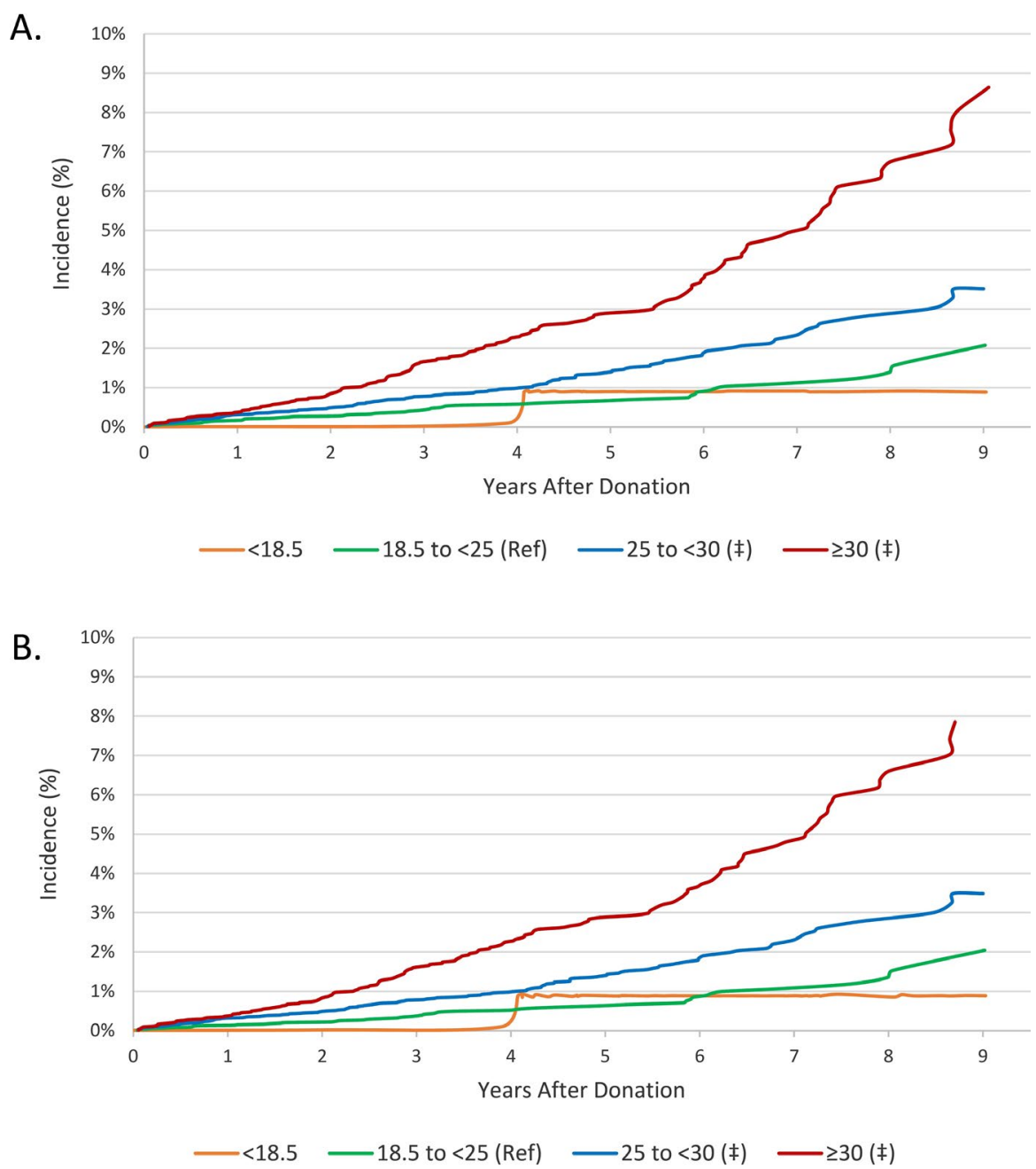

\section{3 | Secondary analysis of weight change after donation}

Among a subgroup of 19528 donors who also had BMI data at the 1-year post-donation anniversary and were free of ADM use in the first year, compared with stable BMI (defined as $\pm 0.5 \mathrm{~kg} / \mathrm{m}^{2}$ change), $\mathrm{BMI}$ increase $>0.5 \mathrm{~kg} / \mathrm{m}^{2}$ by 1 year was associated with increased risk of subsequent ADM use (aHR, $\left.{ }_{1.03} 1.48_{2.14}, P=.036\right)$ after adjustment for all baseline factors including $\mathrm{BMI}$ at donation.

\section{4 | DISCUSSION}

Pharmacy claims can provide efficient, non-obtrusive surrogate measures of treated clinical conditions that can help advance understanding of health outcomes in living donors, an important population that is not followed long-term in the US national transplant registry. To improve understanding of the frequency and correlates of ADM use in living kidney donors, and specifically examine associations of ADM use with BMI, we integrated the national US donor registry with a pharmacy claims database that captures medication fills after donation. This analysis of a large, national donor sample yielded several notable observations: (a) The incidence of ADM use rose in a graded manner with higher $\mathrm{BMI}$ at donation, from $0.9 \%$ in underweight donors to $8.5 \%$ in obese donors by 9 years. (b) Associations of higher baseline BMI with ADM treatments persisted after covariate adjustment, such that obesity was associated with more than four times the likelihood of ADM use. (c) Metformin was the most commonly used ADM after donation, and use was five times more common among obese than among normal weight donors. (d) Overweight and obese BMI were not associated with post-donation insulin use. (e) Use of ADM was more common in recent years. (f) BMI increases over the first year post-donation were also associated with increased risk of subsequent ADM use, independent of BMI at donation.

Our observation of graded increase in ADM use with higher BMI is consistent with prior studies reporting associations of obesity with measures of diabetes mellitus, both in the general population and in living donors. In one study that reported findings from two general population cohorts of 77690 women and 46060 men, higher BMI was associated with increased risk of diabetes at 10 years of followup: compared with normal BMI, BMls of 25 to $<30,30$ to $<35$, and $\geq 35 \mathrm{~kg} / \mathrm{m}^{2}$ were associated with approximately 4,10 , and 20 times higher adjusted risk of diabetes. ${ }^{5} \mathrm{~A}$ single-center US study that included 3752 predominantly white donors (1975-2014, 17\% obese) 
TAB LE 2 Adjusted associations of body mass index (BMI) and other baseline factors with antidiabetic medication use in living kidney donors

\begin{tabular}{|c|c|c|c|}
\hline Characteristics at donation & $\begin{array}{l}\text { Any ADM use } \\
\text { aHR ( } 95 \% \mathrm{CL})\end{array}$ & $\begin{array}{l}\text { Non-insulin } \\
\text { ADM use } \\
\text { aHR ( } 95 \% \mathrm{CL})\end{array}$ & $\begin{array}{l}\text { Insulin use } \\
\text { aHR ( } 95 \% \mathrm{CL})\end{array}$ \\
\hline \multicolumn{4}{|l|}{$\mathrm{BMI}\left(\mathrm{kg} / \mathrm{m}^{2}\right)$} \\
\hline$<18.5$ & $0.63(0.09-4.52)$ & $0.67(0.09-4.85)$ & $\mathrm{N} / \mathrm{A}$ \\
\hline 18.5 to $<25$ & Reference & Reference & Reference \\
\hline 25 to $<30$ & $2.23(1.62-3.06)^{\ddagger}$ & $2.40(1.74-3.33)^{\ddagger}$ & $0.79(0.28-2.22)$ \\
\hline \multicolumn{4}{|l|}{ Age (y) } \\
\hline $18-30$ & $1.18(0.86-1.60)$ & $1.16(0.85-1.58)$ & $1.68(0.55-5.10)$ \\
\hline $31-44$ & Reference & Reference & Reference \\
\hline $45-59$ & $0.80(0.61-1.04)$ & $0.79(0.61-1.04)$ & $1.03(0.38-2.81)$ \\
\hline$\geq 60$ & $0.92(0.59-1.45)$ & $0.96(0.61-1.51)$ & N/A \\
\hline Black & $1.26(0.92-1.73)$ & $1.26(0.91-1.73)$ & $3.48(1.26-9.63)^{*}$ \\
\hline Hispanic & $1.29(0.95-1.77)$ & $1.27(0.92-1.75)$ & $2.91(1.05-8.12)^{*}$ \\
\hline Other & $1.73(1.04-2.89)^{*}$ & $1.79(1.07-2.99)^{*}$ & N/A \\
\hline \multicolumn{4}{|l|}{ Donor/recipient relationship } \\
\hline Related recipient with diabetes & $1.13(0.83-1.54)$ & $1.10(0.80-1.51)$ & $1.46(0.49-4.38)$ \\
\hline Related recipient without diabetes & Reference & Reference & Reference \\
\hline Unrelated & $0.89(0.70-1.13)$ & $0.88(0.69-1.12)$ & $1.03(0.42-2.52)$ \\
\hline \multicolumn{4}{|l|}{ Highest level of education } \\
\hline College \& Higher & $0.99(0.77-1.27)$ & $1.04(0.81-1.33)$ & $0.44(0.19-1.04)$ \\
\hline Grade/High School & Reference & Reference & Reference \\
\hline Insured & Reference & Reference & Reference \\
\hline Uninsured & $0.93(0.64-1.33)$ & $0.96(0.67-1.38)$ & $0.22(0.03-1.68)$ \\
\hline Unknown & $0.74(0.53-1.04)$ & $0.76(0.55-1.07)$ & $0.28(0.06-1.27)$ \\
\hline Hypertension history & $1.26(0.74,2.14)$ & $1.41(0.86,2.33)$ & $5.36(1.49,19.26)^{*}$ \\
\hline Smoking history & $1.03(0.81-1.31)$ & $1.03(0.81-1.32)$ & $1.10(0.45-2.69)$ \\
\hline \multicolumn{4}{|l|}{ eGFR at donation $\left(\mathrm{mL} / \mathrm{min}\right.$ per $\left.1.73 \mathrm{~m}^{2}\right)$} \\
\hline$<60$ & $1.64(0.86-3.13)$ & $1.75(0.94-3.25)$ & $1.77(0.22-13.94)$ \\
\hline 60 to $<90$ & $1.04(0.81-1.34)$ & $1.02(0.80-1.31)$ & $0.80(0.30-2.12)$ \\
\hline$\geq 90$ & Reference & Reference & Reference \\
\hline \multicolumn{4}{|l|}{ Nephrectomy type } \\
\hline Open & $0.87(0.52-1.47)$ & $0.89(0.53-1.50)$ & N/A \\
\hline Laparoscopic/unknown & Reference & Reference & Reference \\
\hline \multicolumn{4}{|l|}{ Year of donation } \\
\hline $2007-2010$ & Reference & Reference & Reference \\
\hline 2011-2013 & $1.41(1.08-1.86)^{*}$ & $1.45(1.10-1.91)^{*}$ & $0.49(0.17-1.46)$ \\
\hline $2014-2016$ & $2.36(1.60-3.48)^{\ddagger}$ & $2.43(1.63-3.62)^{\ddagger}$ & $1.30(0.30-5.67)$ \\
\hline
\end{tabular}

P-value vs reference:

${ }^{*} P<.05-.002$;

${ }^{\dagger} P=.001-.0002$

${ }^{\ddagger} P<.0001$.

ADM, antidiabetic medication; BMI, body mass index; $C L$, confidence limits; eGFR, estimated glomerular filtration rate. 
FIGURE 2 Incidence of antidiabetic medication use according to year of donation

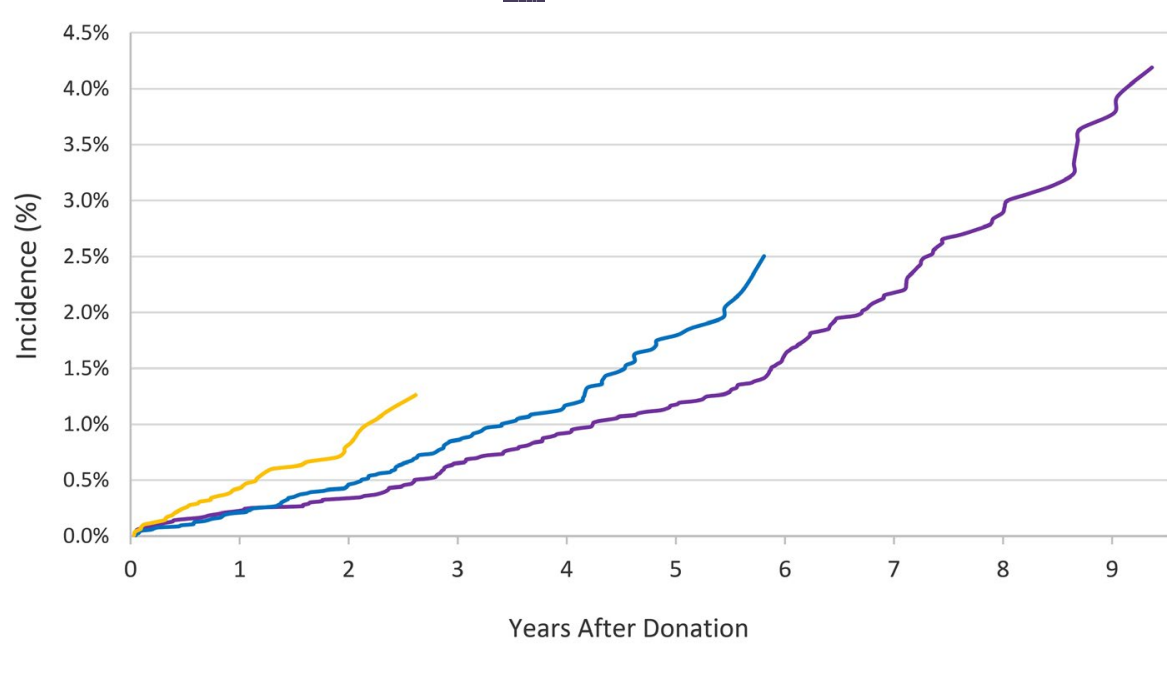

Year of Donation —2007-2010 — 2011-2013 - 2014-2016 found PDDM rates by the end of follow-up in $12 \%$ vs $6 \%$ of donors with baseline $\mathrm{BMI} \geq 30 \mathrm{vs}<30 \mathrm{~kg} / \mathrm{m}^{2}$, and adjusted risk related to obesity was three times that of normal weight. ${ }^{9}$ Another smaller single-center study of 388 donors from Egypt (1976-2014) also found increased PDDM risk with higher BMI (4.1\% in normal weight, $6 \%$ in overweight, and $25 \%$ in obese donors over follow-up to 2014). ${ }^{27}$ The higher incidence of PDDM in these two single-center cohorts likely reflects maximal follow-up over decades. Our study expands upon these single-center reports by examining a national US cohort and using $A D M$ as a non-obtrusive measure of a clinical condition that does not require return to the center for ascertainment.

The racial disparity in obesity observed in our study sample is present in the general population and was previously reported among both the general population ${ }^{28}$ and living donors. ${ }^{18}$ We previously identified racial and ethnic disparity in PDDM ascertained by diagnostic codes on medical claims; black and Hispanic donors had 1.52 and 1.65 times the likelihood of PDDM compared with white donors, respectively, based on clinical diagnoses after an average 7.7 years of follow-up. ${ }^{29}$ That sample was limited to 4650 privately insured donors, and importantly, baseline BMI was not available. In the current study, black race, and Hispanic ethnicity were not significantly associated with ADM treatments overall, which may reflect the impact of adjustment for BMI (given the higher prevalence of obesity in these racial/ethnic groups), difference in the study samples, or the impact of using treatment compared with billing claims as the outcome measure. However, black and Hispanic donors had 3.5 and 2.9 times the likelihood of insulin use, respectively, compared with white donors in the current study, perhaps reflecting a greater severity of PDDM. Our finding of increased risk of ADM use for women in the multivariate model contrasts with the increased risk for men reported in the large single-center study of white donors, which may reflect differences in the outcome, and possibly care seeking for treatment. ${ }^{30}$
FIGURE 3 Distribution of antidiabetic medication categories filled by 9 y postdonation, according to body mass index at donation. Medication categories are not mutually exclusive

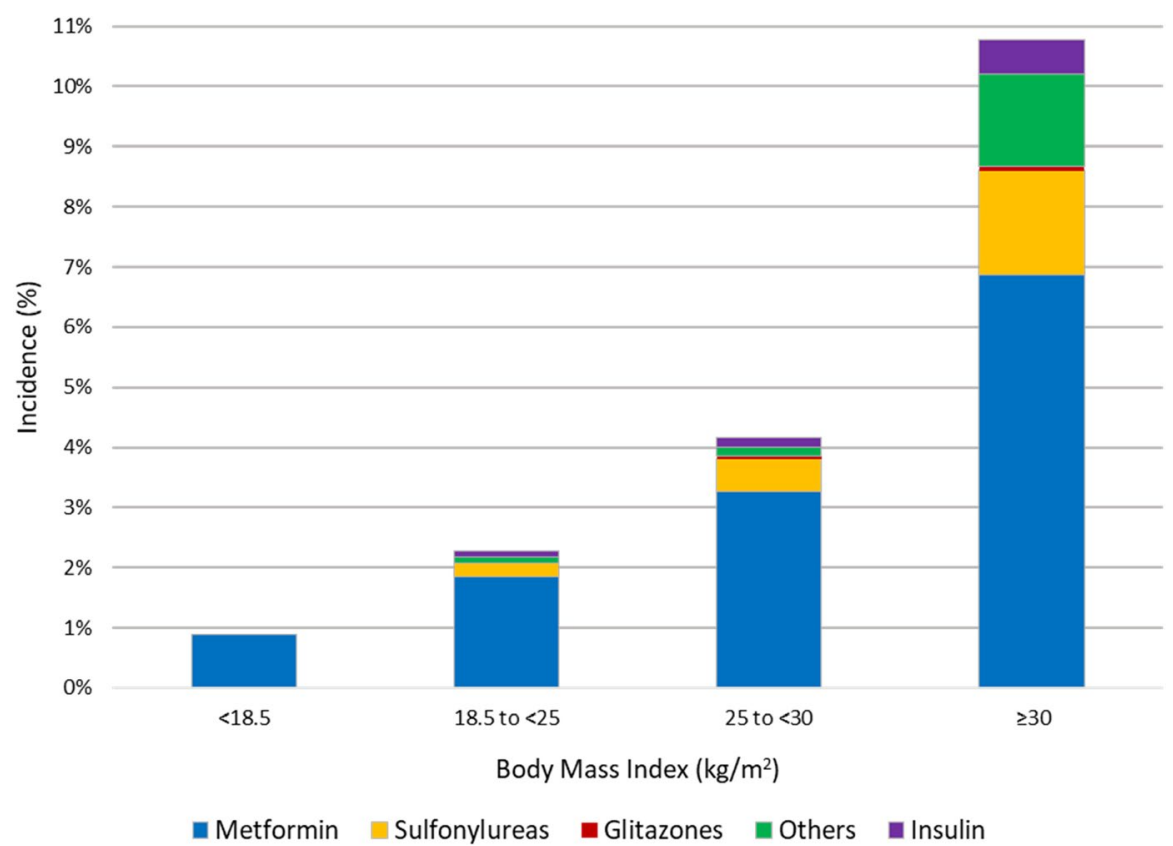




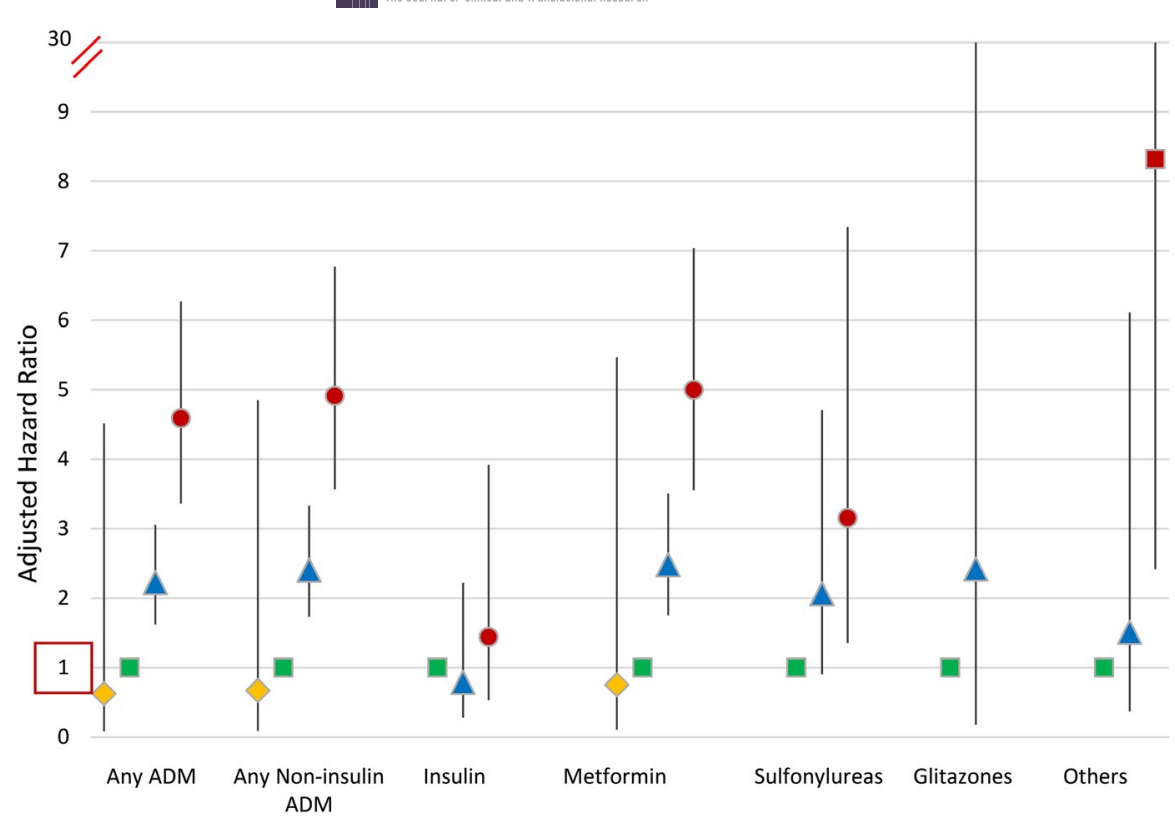

FIGURE 4 Adjusted associations of body mass index (BMI) at donation with antidiabetic medication use categories after donation. Adjusted for other baseline factors in Table 1
The temporal increase in ADM use observed in the current study warrants attention. While the 2017 diabetes mellitus report card by the Centers for Disease Control and Prevention noted that the annual incidence of diabetes in the US peaked in 2008 at $0.85 \%$ and then declined to $0.65 \%$ in $2015,{ }^{31}$ use of oral antidiabetic medications appears to be rising worldwide. ${ }^{32}$ Although reasons for the temporal trend in ADM use in living donors are not known, liberalization in accepting donors with metabolic abnormalities, including prediabetes, in more recent years could be contributing. ${ }^{18,33}$ One study of 8951 donors from three US centers (1963 and 2007) reported that median values of fasting glucose at donation steadily increased over time. ${ }^{33}$ Notably, wide acceptance of hemoglobin A1c in place of oral glucose tolerance testing as one of the diagnostic criteria for diabetes around 2011 may also have contributed to this observed trend. ${ }^{34}$ Future work should monitor ongoing trends in ADM use and other measures of PDDM, and associations with baseline factors.

Metformin is the first-line pharmaceutical treatment for diabetes mellitus because of its efficacy in glucose control, lower risk of adverse effect profile, such as weight gain and hypoglycemia, and lower costs. ${ }^{35}$ Beginning diabetes treatment with metformin has also been associated with reduced subsequent need for treatment intensification. ${ }^{36}$ However, metformin has been linked to rare but fatal lactic acidosis complications, especially in patients with eGFR $<30 \mathrm{~mL} / \mathrm{min}$ per $1.73 \mathrm{~m}^{2}$, which has led the US Food and Drug Administration (FDA) to label it as contraindicated for such patients. Since most donors are expected to have eGFR levels $>30 \mathrm{~mL} / \mathrm{min}$ per $1.73 \mathrm{~m}^{2}$, use is likely safe and appropriate, but notably, concern for reduced kidney function was not a deterrent to use, in contrast with infrequent use in the transplant population at higher eGFR levels. ${ }^{19}$ In the context of obesity, even independent of diabetes, metformin may have benefit for weight loss. In a randomized placebo-controlled study of adults (BMI $\geq 24 \mathrm{~kg} / \mathrm{m}^{2}$ and prediabetes), metformin was associated with $3.5 \%$ weight loss over 9 years of follow-up. ${ }^{37}$ However, the current FDA labeled indication for metformin is type 2 diabetes alone, and weight loss and prediabetes are not approved indications. Thus, while it is possible that our study measure of ADM use captured indications other than type 2 diabetes (such as off-label, use or uncommon treatment of polycystic ovarian syndrome) type 2 diabetes is the primary prescribing indication for metformin.

While ongoing work is needed, our study has implications for living donor care. Given the recently identified associations of obesity with post-donation ESRD, ${ }^{2}$ obesity should be considered a renal risk factor in donors, and of particular importance because of potential modifiability. Lifestyle factors that contribute to obesity include low physical activity, high caloric intake, and poor sleep quality. ${ }^{38}$ Interventional data among donors are lacking, but previous studies have shown that weight loss among obese adults is associated with reduced risk of diabetes which, in turn, would translate into reduced risk of ESRD. In a general population study of 114281 women (1976-1990), the more weight gain during follow-up, the higher the risk of diabetes; in contrast, women who lost $>5 \mathrm{~kg}$ had reduced risk of diabetes. ${ }^{39}$ However, achieving and sustaining weight loss is not trivial. Issa et al ${ }^{40}$ reported that only $16 \%$ $(14 / 90)$ of high BMI $\left(>30 \mathrm{~kg} / \mathrm{m}^{2}\right)$ donors at one center were able to achieve weight loss to a BMI of $<30 \mathrm{~kg} / \mathrm{m}^{2}$ prior to donation. At 15 years of follow-up, nearly all the high BMI donors (12/14) who had lost weight pre-donation gained weight, with a mean increase in BMI of $4.8 .^{40}$ In our subgroup analysis of donors who also had $\mathrm{BMI}$ data at the 1-year post-donation anniversary, compared with stable BMI, BMI increase $>0.5 \mathrm{~kg} / \mathrm{m}^{2}$ by year 1 was associated with increased risk of subsequent ADM use. This is consistent with recent data that post-donation weight gain is associated with higher risk of diabetes. ${ }^{41}$ Although prospective evaluations of dietary interventions and monitored exercise programs in donors are needed to advance effective care, donor candidates should be counseled on lifestyle interventions to support achievement and maintenance of healthy body weight, and regular exercise according to guidelines for the general population. ${ }^{1}$ Importantly, these interventions 
should be initiated before donation and maintained lifelong, including as part of annual post-donation follow-up. ${ }^{1}$

There are several strengths to our study. We used pharmacy claims as a measure of diabetes, a non-obtrusive surrogate that avoids recall bias associated with survey studies, and complements information acquired though other study designs. Using this large, diverse national dataset, we confirmed associations of obesity at the time of donation with increased incidence of PDDM, previously reported among a single-center sample of predominantly white donors. ${ }^{9}$ We also found that black and Hispanic donors, compared with white donors, had higher risk of insulin use after donation. We also observed that donation to a related recipient with diabetes was associated with a trend toward higher ADM use, but the association was not statistically significant. Future work is needed to define the impacts of family history of diabetes, race, and genetics in the complex relationships of obesity, diabetes, and post-donation ESRD.

Our study has limitations. Linked pharmacy data were available for $49 \%$ of US donors in the study period; thus, while our national study is larger than prior single-center cohorts, results may not generalize beyond the study sample. However, baseline characteristics of the sample were similar to those of all US donors. We also lacked data for comparisons to healthy nondonors, and focused on comparisons of donor subgroups. ${ }^{42}$ Our measure was based on pharmacy fills, and we lacked laboratory tests for confirmation, such as hemoglobin A1c, fasting glucose, or oral glucose tolerance testing. However, our inability to capture diabetes controlled by lifestyle measures should under-estimate the risk associated with obesity in donors. As discussed above, off-label prescribing of metformin may be captured as part of ADM use. We lacked data on fat distribution, which may be a stronger predictor for risk of ADM use than BMI. We were able to identify donation to related recipients with diabetes mellitus, but the registry does not include information on diabetes status in other relatives, or family history information for unrelated donors, or lifestyle risk factors. We lacked information on BMI changes for the entire follow-up period. However, in a subset with BMI data available at 1-year anniversary, we found that BMI increase is associated with subsequent $A D M$ use, independent of BMI at donation.

In conclusion, linkage of national US donor registry data with pharmacy fill records demonstrates that being overweight or obese at the time of living kidney donation is strongly associated with ADM use after donation. In addition, the risk of ADM use appeared to increase over the period of our study. While this study did not assess the impact of donation on the development of obesity, these data advance understanding of the relationship obesity, when present among donors, with subsequent ADM use. Future work should monitor ongoing trends in ADM use and other measures of diabetes, associations with baseline factors including BMI and metabolic profile, and impact on renal and cardiovascular outcomes among living donors. All stakeholders-including patients, primary physicians, transplant programs, policy makers, insurers, and researchers-should recognize the importance of obesity as a contributor to post-donation health and work together to develop resources and interventions to support optimal donor outcomes.

\section{ACKNOWLEDGEMENTS}

The authors thank SRTR colleague Nan Booth, MSW, MPH, ELS, for manuscript editing. An abstract describing portions of this work was an "Abstract of Distinction" at the American Transplant Congress 2017, Chicago, IL, and highlighted by Renal \& Urology News, https://bit.ly/2pmCvFj.

\section{CONFLICT OF INTEREST}

The authors declare no conflicts of interest.

\section{AUTHORS' CONTRIBUTIONS}

KLL and MAS participated in study design, acquisition of data and regulatory approvals, data analysis, and writing of the paper. FMK, NS, ASN, NNL, AXG, DA, GPS, BLK, DLS, MLH, ABM and CHM participated in study design, interpretation, and writing of the paper as part of our pharmacoepidemiology consortium. ZZ Participated in data analysis and manuscript preparation.

\section{ORCID}

Krista L. Lentine (iD https://orcid.org/0000-0002-9423-4849

Nagaraju Sarabu iD https://orcid.org/0000-0001-6084-8677

Abhijit S. Naik iD https://orcid.org/0000-0003-1602-9425

David Axelrod (iD https://orcid.org/0000-0001-5684-0613

Zidong Zhang (iD https://orcid.org/0000-0002-1084-9732

Gregory P. Hess iD https://orcid.org/0000-0001-8942-6865

Macey L. Henderson iD https://orcid.org/0000-0002-4239-1252

Courtenay M. Holscher iD https://orcid.org/0000-0002-5808-5954

\section{REFERENCES}

1. Lentine KL, Kasiske BL, Levey AS, et al. KDIGO clinical practice guideline on the evaluation and care of living kidney donors. Transplantation. 2017;101(8S Suppl 1):S1-S109.

2. Anjum S, Muzaale AD, Massie AB, et al. Patterns of end-stage renal disease caused by diabetes, hypertension, and glomerulonephritis in live kidney donors. Am J Transplant. 2016;16(12):3540-3547.

3. Ibrahim HN, Foley RN, Reule SA, et al. Renal function profile in white kidney donors: the first 4 decades. J Am Soc Nephrol. 2016;27(9):2885-2893.

4. OPTN (Organ Procurement and Transplantation Network)/UNOS (United Network for Organ Sharing). OPTN Policies, Policy 14: Living Donation. http://optn.transplant.hrsa.gov/governance/polic ies/. Accessed July 7, 2019.

5. Field AE, Coakley EH, Must A, et al. Impact of overweight on the risk of developing common chronic diseases during a 10-year period. Arch Intern Med. 2001;161(13):1581-1586.

6. Hsu CY, McCulloch CE, Iribarren C, Darbinian J, Go AS. Body mass index and risk for end-stage renal disease. Ann Intern Med. 2006;144(1):21-28.

7. Grams ME, Sang Y, Levey AS, et al. Kidney-failure risk projection for the living kidney-donor candidate. New Engl J Med. 2016;374(5):411-421. 
8. Locke JE, Reed RD, Massie A, et al. Obesity increases the risk of end-stage renal disease among living kidney donors. Kidney Int. 2017;91(3):699-703.

9. Serrano OK, Sengupta B, Bangdiwala A, et al. Implications of excess weight on kidney donation: long-term consequences of donor nephrectomy in obese donors. Surgery. 2018;164(5):1071-1076.

10. Delmonico F. A report of the amsterdam forum on the care of the live kidney donor: data and medical guidelines. Transplantation. 2005;79(6 Suppl):S53-66.

11. The British Transplant Society and The Renal Association. The United Kingdom Guidelines for Living Donor Kidney Transplantation. 4th ed., 2018.

12. Abramowicz D, Cochat $\mathrm{P}, \mathrm{Claas} \mathrm{FH}$, et al. European Renal Best Practice Guideline on kidney donor and recipient evaluation and perioperative care. Nephrol Dial Transplant. 2015;30(11):1790-1797.

13. Spanish Society of Nephrology (S.E.N.) and Spanish Transplant Organisation (ONT). Recommendations for living-donor kidney transplantation. Nefrologia. 2010;30(Suppl 2):1-105.

14. AST Live Donor Community of Practice. Live Kidney Donor Medical Toolkit for Medical Providers. The Obese Kidney Donor. https:// www.myast.org/patient-information/live-donor-toolkit. Accessed July 7, 2019.

15. Pascual J, Abramowicz D, Cochat P, et al. European renal best practice guideline on the management and evaluation of the kidney donor and recipient. Nefrologia. 2014;34(3):293-301.

16. Isbel N. The CARI guidelines. Donors at risk: obesity. Nephrology (Carlton). 2010;15(Suppl 1):S121-132.

17. Ethics Committee of the Transplantation Society. The consensus statement of the Amsterdam Forum on the Care of the Live Kidney Donor. Transplantation. 2004;78(4):491-492.

18. Naik AS, Cibrik DM, Sakhuja A, et al. Temporal trends, centerlevel variation, and the impact of prevalent state obesity rates on acceptance of obese living kidney donors. Am J Transplant. 2018;18(3):642-649.

19. Vest LS, Koraishy FM, Zhang Z, et al. Metformin use in the first year after kidney transplant, correlates, and associated outcomes in diabetic transplant recipients: a retrospective analysis of integrated registry and pharmacy claims data. Clin Transplant. 2018;32(8):e13302.

20. Lentine KL, Schnitzler MA, Garg AX, et al. Understanding antihypertensive medication use after living kidney donation through linked national registry and pharmacy claims data. Am J Nephrol. 2014;40(2):174-183.

21. Lentine KL, Lam NN, Schnitzler MA, et al. Gender differences in use of prescription narcotic medications among living kidney donors. Clin Transplant. 2015;29(10):927-937.

22. Lentine KL, Lam NN, Schnitzler MA, et al. Predonation prescription opioid use: a novel risk factor for readmission after living kidney donation. Am J Transplant. 2017;17(3):744-753.

23. Lam NN, Garg AX, Segev DL, et al. Gout after living kidney donation: correlations with demographic traits and renal complications. Am J Nephrol. 2015;41(3):231-240.

24. Lam NN, McArthur E, Kim SJ, et al. Gout after living kidney donation: a matched cohort study. Am J Kidney Dis. 2015;65(6):925-932.

25. Lentine KL, Segev DL. Better understanding live donor risk through big data. Clin J Am Soc Nephrol. 2013;8(10):1645-1647.

26. Lentine KL, Yuan H, Tuttle-Newhall JE, et al. Quantifying prognostic impact of prescription opioid use before kidney transplantation through linked registry and pharmaceutical claims data. Transplantation. 2015;99(1):187-196.

27. Abuelmagd MM, Nagib AM, Abuelmagd MM, et al. Study of the risk factors and complications of diabetes mellitus after live kidney donation. Transplant Proc. 2015;47(4):1152-1157.
28. Center For Disease Control. Prevalence of Obesity Among Adults and Youth: United States, 2015-2016. 2017;2018(December 18) https://www.cdc.gov/nchs/data/databriefs/db288.pdf. Accessed July 7, 2019.

29. Lentine $\mathrm{KL}$, Schnitzler MA, Xiao $\mathrm{H}$, et al. Racial variation in medical outcomes among living kidney donors. N Engl J Med. 2010;363(8):724-732.

30. Ibrahim HN, Kukla A, Cordner G, Bailey R, Gillingham K, Matas AJ. Diabetes after kidney donation. Am J Transplant. 2010;10(2):331-337.

31. Centers for Disease Control and Prevention. Diabetes Report Card 2017. https://www.cdc.gov/diabetes/pdfs/library/diabetesreportc ard2017-508.pdf. Accessed July 7, 2019.

32. Fazeli Farsani S, Souverein PC, Overbeek JA, et al. Long term trends in oral antidiabetic drug use among children and adolescents in the Netherlands. Br J Clin Pharmacol. 2015;80(2):294-303.

33. Taler SJ, Messersmith EE, Leichtman AB, et al. Demographic, metabolic, and blood pressure characteristics of living kidney donors spanning five decades. Am J Transplant. 2013;13(2):390-398.

34. American Diabetes Association. Diagnosis and classification of diabetes mellitus. Diabetes Care. 2012;35(Suppl 1):S64-71.

35. Inzucchi SE, Bergenstal RM, Buse JB, et al. Management of hyperglycemia in type 2 diabetes, 2015: a patient-centered approach: update to a position statement of the American Diabetes Association and the European Association for the Study of Diabetes. Diabetes Care. 2015;38(1):140-149.

36. Berkowitz SA, Krumme AA, Avorn J, et al. Initial choice of oral glucose-lowering medication for diabetes mellitus: a patientcentered comparative effectiveness study. JAMA Intern Med. 2014;174(12):1955-1962.

37. Diabetes Prevention Program Research Group. Long-term safety, tolerability, and weight loss associated with metformin in the Diabetes Prevention Program Outcomes Study. Diabetes Care. 2012;35(4):731-737.

38. Padwal RS. Obesity, diabetes, and the metabolic syndrome: the global scourge. Can J Cardiol. 2014;30(5):467-472.

39. Colditz GA, Willett WC, Rotnitzky A, Manson JE. Weight gain as a risk factor for clinical diabetes mellitus in women. Ann Intern Med. 1995;122(7):481-486.

40. Issa N, Askandarani S, Gillingham K, et al. Fate of living kidney donors who lost weight to become donors. (Abstract \# A331). Transplantation. 2014;98(Supplement 1):494.

41. Issa N, Sánchez OA, Kukla A, et al. Weight gain after kidney donation: Association with increased risks of type 2 diabetes and hypertension. Clin Transplant. 2018;32(9):e13360.

42. Lentine KL, Segev DL. Understanding and communicating medical risks for living kidney donors: a matter of perspective. J Am Soc Nephrol. 2017;28(1):12-24.

\section{SUPPORTING INFORMATION}

Additional supporting information may be found online in the Supporting Information section at the end of the article.

How to cite this article: Lentine KL, Koraishy FM, Sarabu N, et al. Associations of obesity with antidiabetic medication use after living kidney donation: An analysis of linked national registry and pharmacy fill records. Clin Transplant.

2019;33:e13696. https://doi.org/10.1111/ctr.13696 